Neurosurg Focus 24 (5):E11, 2008

\title{
The principles of skull base radiosurgery
}

\author{
Douglas Kondziolka, M.D., F.R.C.S.C., ${ }^{1}$ John C. Flickinger, M.D., ${ }^{2}$ \\ AND L. DADE LUNSFORD, M.D. ${ }^{1}$ \\ Departments of ${ }^{\prime}$ Neurological Surgery and ${ }^{2}$ Radiation Oncology, University of Pittsburgh, \\ Pennsylvania
}

\begin{abstract}
$\checkmark$ Stereotactic radiosurgery is commonly used for selected patients with benign cranial base tumors. The goal of radiosurgery is cessation of tumor growth and preservation of neurological function. Over the last 2 decades, the technique of radiosurgery has evolved due to improved imaging, better radiosurgical devices and software, and the continued analysis of results. In this report, the authors discuss technical concepts and dose selection in skull base radiosurgery.(DOI: $10.3171 /$ FOC/2008/24/5/E11)
\end{abstract}

\section{KEY WORDS • meningioma • radiation dose • radiosurgery • schwannoma • skull base}

$\mathrm{M}$ OST patients who undergo SRS at the skull base have a benign tumor such as a schwannoma, meningioma, or pituitary adenoma. Such patients are expected to live for an extended period. Thus, practitioners must strive for long-term tumor control after radiosurgery, and the morbidity profile must be low. Effective radiosurgery is based on the principles of specificity and selectivity. ${ }^{11}$ Precision and conformal dose planning allow specific target irradiation with a steep falloff into other surrounding structures. Selectivity refers to the biological differences in the response of different tissues.

Within and adjacent to the CS region, the intimate association of the tumor with critical neurovascular structures makes the resection difficult. Numerous studies demonstrate that SRS provides effective tumor control with minimal morbidity. Its success with respect to meningiomas of the CS is measured by its long-term tumor control rate, and its success with respect to pituitary adenomas is measured by both endocrinological control and arrest of tumor growth. At our center we used and evaluated GKS for a variety of benign, extraaxial basal tumors. These included schwannomas, meningiomas, hemangiomas, pituitary adenomas, craniopharyngiomas, and selected malignant neoplasms such as chordomas and chondrosarcomas.

Abbreviations used in this paper: $\mathrm{CS}=$ cavernous sinus; GKS = Gamma Knife surgery; MR = magnetic resonance; SRS = stereotactic radiosurgery; SRT = stereotactic radiotherapy; VS = vestibular schwannoma.

\section{Technique}

The first step in performing radiosurgery is proper patient selection. Patients referred to our center for radiosurgery are reviewed by neurosurgeons with experience in both microsurgical resection and radiosurgery. Patients with large tumors associated with neurological compromise are advised to undergo microsurgical resection of their tumors to relieve mass effect more rapidly. In contrast, elderly patients with an incidentally discovered tumor may be advised to undergo a period of observation. The decision to perform a radiosurgery procedure must include an estimation of the final dose plan. Questions that must be asked include the following. 1) Can an effective dose be delivered safely? 2) Is the tumor volume too large? 3) Is the imaging consistent with the presumed diagnosis if there is no prior biopsy? 4) Will the procedure meet the patient's goals?

The primary imaging sequence we use is a volume acquisition, spoiled gradient-recalled acquisition in the steady state technique performed in the axial plane. Fatsuppression images are used for tumors adjacent to the orbit or infratemporal fossa, or in other cases in which fat was placed at surgery. Images were spaced from 1 to $2 \mathrm{~mm}$ apart. The goal of dose planning is to create a conformal isodose configuration that completely covers the tumor, with a minimal amount of radiation being delivered to the surrounding structures. This is particularly important for cranial base tumors because the regional anatomy typically includes cranial nerves, the brainstem, small or large vessels, venous sinuses, or the cochlea. Using a combination of multiple irradiation isocenters of different size, differential weighting of the individual isocenters, and selective 
D. Kondziolka, J. C. Flickinger, and L. D. Lunsford

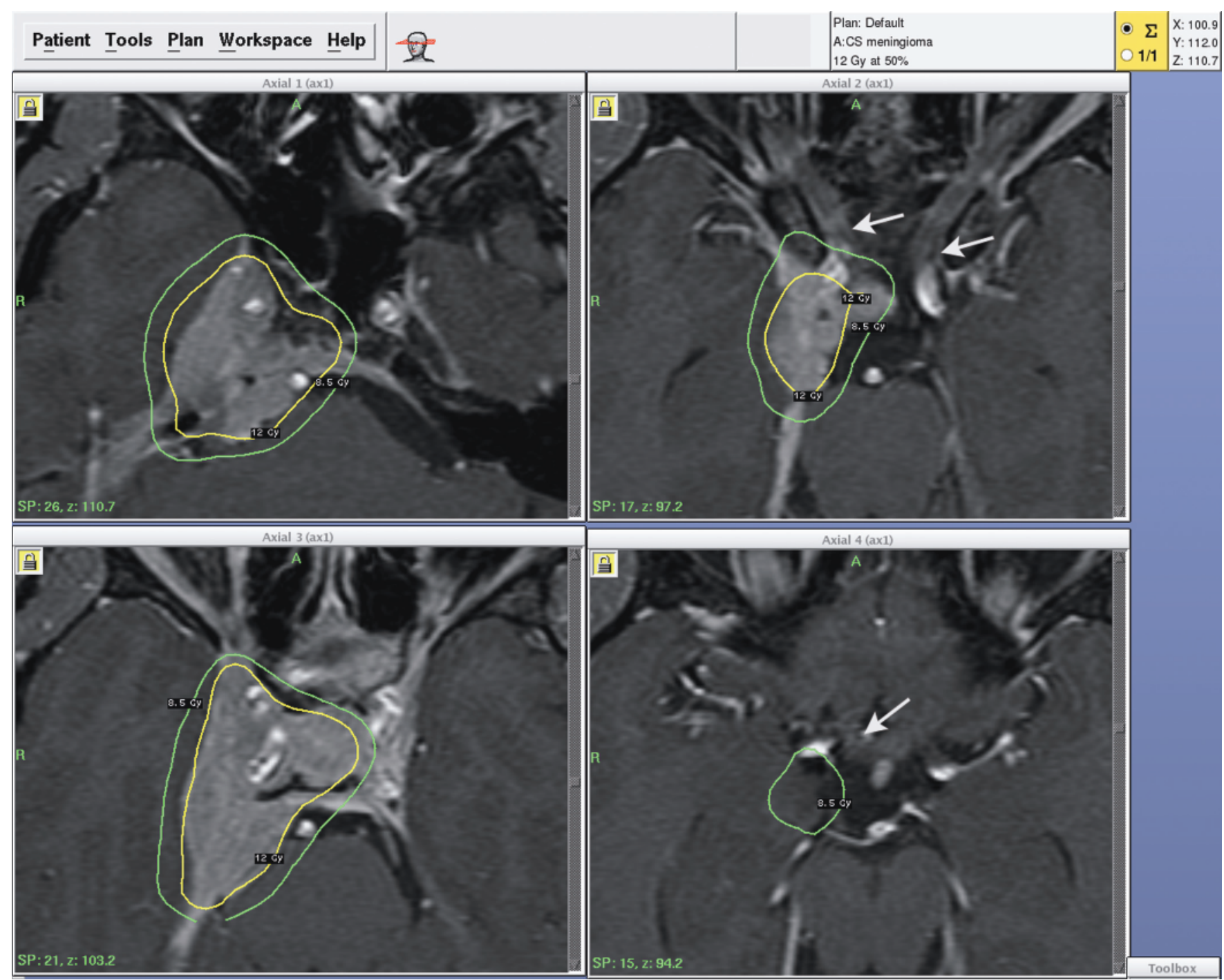

FIG. 1. Stereotactic MR imaging-based GKS dose plan for a patient with a right CS meningioma located close to the optic nerves and chiasm (arrows). The tumor was irradiated to a margin dose of 12 Gy (yellow line), with a maximum dose of 24 Gy. The 8.5-Gy dose line (green line) is shown and remains outside the optic nerves.

blocking of the collimated beams of radiation, it is possible to produce dose plans that closely conform to the irregular shape of all tumors. The Perfexion model Gamma Knife efficiently allows beam sector blocking and the simultaneous use of different collimator sizes, radically increasing the options available for dose planning. Sector blocking with the Perfexion unit is much easier than beam blocking with the 4C unit or other earlier Gamma Knife models. With those models, however, beams are blocked individually. Although this does provide more options from a practical perspective, beam blocking is now performed more often with the Perfexion unit.

To create a plan for an irregularly shaped tumor, small collimators (4 and $8 \mathrm{~mm}$ ) are most useful. Such collimators also offer a steeper falloff in the radiation dose. Thus, in the auditory canal only the smallest beams should be used. Similarly, for that portion of a CS tumor near the optic nerve or chiasm, smaller beams with or without blocking allow effective target irradiation while minimizing regional irradiation. The actual dose selection is based on a number of factors. In the past, dose-prescription formulae, such as the integrated logistic formula for predicting radiation-related complications after radiosurgery, were used for intraparenchymal radiosurgery. ${ }^{2}$ These rules do not really apply for extraaxial basal tumors. Although it appears that some cranial nerves may tolerate high doses of radiation (60-70 Gy) when delivered in multiple fractions, their susceptibilities to injury after single-fraction, high-dose radiation are different. For example, years ago we observed trigeminal and facial neuropathy rates of $\sim 30 \%$ after radiosurgery for VS when the maximum radiation dose was 36-40 Gy. ${ }^{3}$ Different classes of cranial nerves appear to have distinct tolerances for radiation. ${ }^{12}$ Special sensory nerves such as the optic nerve may not tolerate doses $>10-12 \mathrm{~Gy}$, whereas general somatic nerves such as the oculomotor nerve do not appear to be adversely affected by similar doses. 


\section{The CS Region}

Criteria for radiosurgery in and around the CS and parasellar region have been focused mainly on visual safety. It must be remembered that the original concept of " 8 Gy or less" to the optic nerves or chiasm was based on very few data. The original observations were made in a few patients who had computed tomography-based planning (where the chiasm was poorly seen) and where a dose $>11$ or 12 Gy was used..$^{15}$ At the same time, some groups have used doses such as 13 or 14 Gy for patients with craniopharyngiomas that directly compress the optic chiasm, and noted little if any morbidity. In 1991 we made a decision to attempt to eliminate the risk of blindness or a significant visual field deficit by using the 8-Gy dose. At present, we aim to restrict the dose to the optic apparatus to $<8.5 \mathrm{~Gy}$ (Fig. 1). Within the CS, we noted that when exposed to the same high, single-fraction dose, the trigeminal nerve was more susceptible to adverse radiation effects than the oculomotor nerves. Our cumulative experience supports the concept that the single-fraction optic nerve tolerance is 8-10 Gy, depending on the volume of nerve involved.

Leber et al. ${ }^{7}$ found that when the maximum radiation dose to the visual pathways was $<10 \mathrm{~Gy}$, no signs of radiation-induced optic neuropathy were observed. However, when the dose varied from 10 to $<15 \mathrm{~Gy}$, the incidence of radiation-induced optic neuropathy was $26.7 \%$. When the dose was $15 \mathrm{~Gy}$ or more, the incidence was $77.8 \%$. Morita et al..$^{12}$ adopted a policy that allowed short segments of visual pathways to be treated with 12-16 Gy, which the structures tolerated. At our center, by using tumor margin doses of $13 \mathrm{~Gy}$, we achieved long-term growth control and preserved neurological function in most patients with CS meningiomas. Primary radiosurgery provided an actuarial tumor control rate of $96.9 \pm 3 \%$ at 5 and 10 years. ${ }^{8}$ The overall actuarial tumor control rate, excluding malignant or atypical meningiomas, was $93.1 \pm 3.3 \%$ at 5 and 10 years (these rates are expressed as the mean \pm standard deviation). By keeping the optic nerve dose $<8$ Gy and by using a tumor margin dose of $13 \mathrm{~Gy}$, we kept the risk of adverse radiation effects to $6.7 \%$. In the past 7 years, only $1(1.3 \%)$ of 78 patients developed optic neuropathy.

The radiosurgical parameters and dose selection for patients with nonsecreting pituitary tumors are similar to those for patients with meningiomas. For individuals with secretory tumors, we aim to deliver as high a radiosurgery dose as possible because the goal is not only control of the mass, but cessation of protein synthesis. Dose depends on adenoma size, location (for example, proximity to the optic apparatus), and history of radiation therapy. In a recent review, the mean tumor margin dose for all adenomas was $16.2 \mathrm{~Gy}$, but the dose to the margin ranged between 10 and $34 \mathrm{~Gy}$. The mean dose to the optic apparatus was $7 \mathrm{~Gy}$. Despite these efforts, 2 patients treated in our series who received a radiation dose of $<8$ Gy to the optic apparatus and who had appropriate collimator blocking experienced a decline in visual function. One of these patients had tumor growth on follow-up imaging, and the visual decline in this patient was more likely a result of tumor growth and optic apparatus compression than radiation-induced injury. Despite imaging-documented tumor regression, the other patient developed a functional decline in the temporal field of his right eye 7 months after radiosurgery.
Vascular structures and cranial nerves within the CS appear to be less susceptible to adverse radiation effects. More radiation can therefore be delivered for laterally extending adenomas than for suprasellar tumors near the optic apparatus. A rare case of cerebral infarction and internal carotid artery occlusion was reported in 1 patient following GKS for his pituitary adenoma. ${ }^{9}$ We have seen no evidence for vascular injury after GKS at our center.

Endocrinopathies can occur after GKS. ${ }^{6,14}$ While dose planning is done, attempts should be made to avoid excess radiation exposure to the pituitary stalk and obviously normal pituitary gland. ${ }^{4}$ There are no good data, however, on radiation tolerance for the gland.

\section{The Brainstem}

The management of basal tumors that are adjacent to or indent the brainstem is complex due to the location and sensitivity of nearby neurovascular structures. Preservation of brainstem function is more easily achieved when there is space between the tumor and lateral brainstem surface. For lesions that compress the brainstem, many surgeons still favor brainstem decompression. We agree with this approach if there are significant symptoms that must be addressed quickly. If not, many patients will do well after radiosurgery as time passes and the tumor regresses slowly off the brainstem surface. Such tumor regression is most common with schwannomas. ${ }^{3,5,13}$ At present, there is no consensus on dose selection for brainstem tolerance. We teach that a tumor margin dose of $\leq 15$ Gy is reasonable for brainstem radiosurgery. In certain situations a higher dose may be necessary, as is required in radiosurgery performed to treat arteriovenous malformations, for the desired obliteration response.

\section{The Cerebellopontine Angle Region}

Radiosurgery is presently a well-established alternative to microsurgical resection of a VS, and at present may be the most common management option for this tumor. Many patients prefer radiosurgery to resection because of lower morbidity associated with the procedure and similar rates of long-term tumor control. Although comparatively lower than with microsurgery, we reported significant rates of subsequent mild facial weakness $(21 \%)$, facial numbness $(27 \%)$, and decreased hearing (49\%) in our first 5 years of experience with radiosurgery for VS, in which we used tumor margin doses on the order of 16 Gy. Since 1992, most centers have reduced tumor margin dose prescriptions to reduce complications. ${ }^{3}$ Dose planning must use high-resolution stereotactic MR imaging to evaluate tumor properly within the auditory canal, and to identify inner ear structures. Again, small beam diameters are most frequently used. With the Gamma Knife technique, only the 4-mm collimator is used in the auditory canal. Although the 50\% isodose line is most commonly used, the 55 or $60 \%$ lines may be advantageous for some patients if they are well matched to the tumor border. Anatomically, the tumor margin isodose line is kept extremely tight along the anterior and medial tumor surfaces, where the facial and cochlear nerves would be expected. The number of isocenters used can vary from 3 to 20 or so depending on tumor size and configuration. 


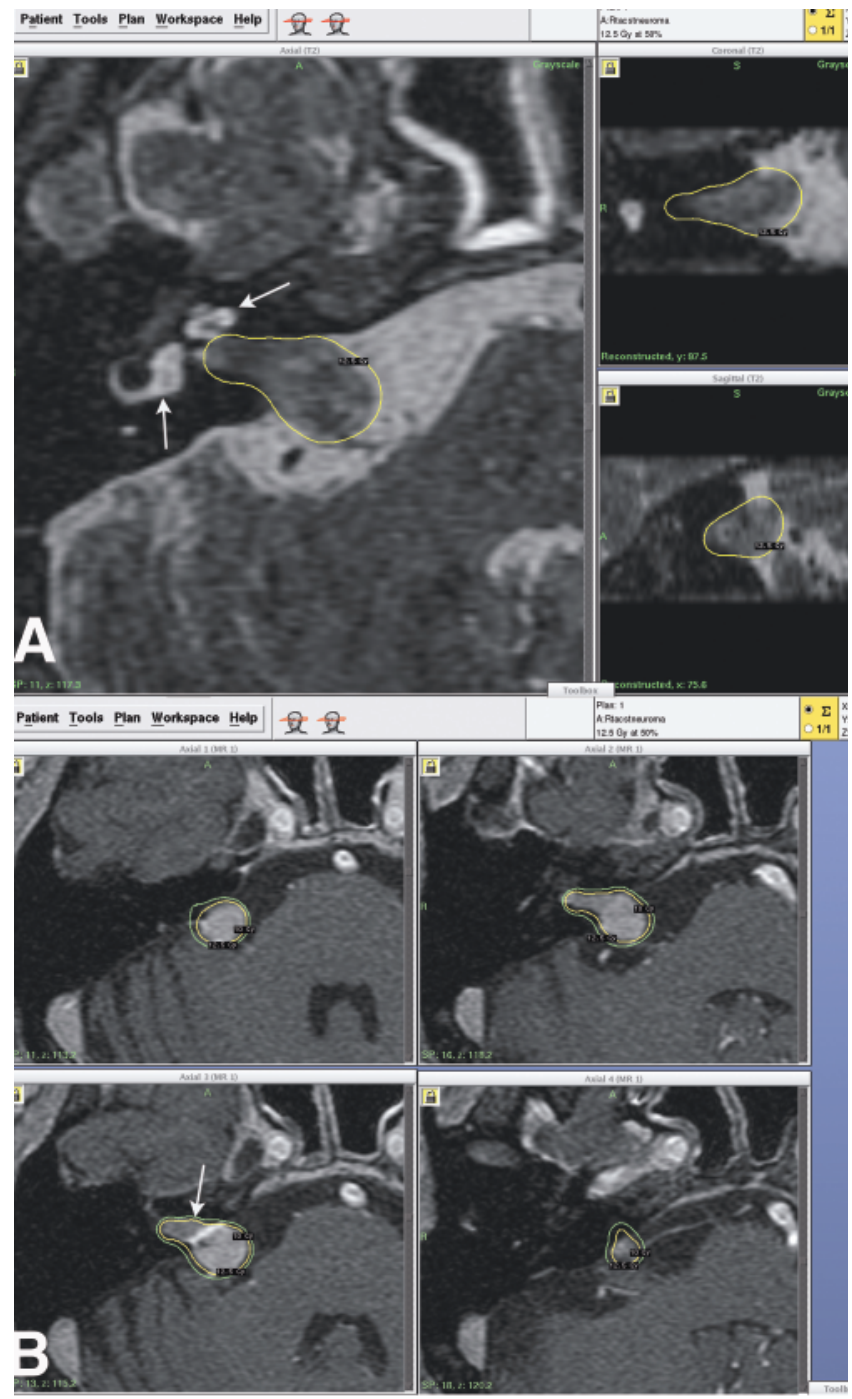

FIG. 2. Stereotactic MR imaging-based GKS dose plans. A: Dose plan for a patient with a VS (long relaxation time MR imaging sequence). The cochlea and horizontal semicircular canal (arrows) are identified lateral to the internal auditory canal fundus. The tumor margin dose was $12.5 \mathrm{~Gy}$ (yellow lines). B: On the contrast-enhanced axial MR imaging sequences, a conformal plan is created from the inferior to superior extent of the tumor. The facial and cochlear nerves would be expected to course along the anterior tumor surface (arrow).

Currently, most centers recommend a tumor margin dose of $12-13 \mathrm{~Gy} .{ }^{3}$ Ten or $11 \mathrm{~Gy}$ has been used by some groups for larger tumors, although we are concerned that this dose may be too low. In patients in whom hearing preservation is the goal, we use 12.5 Gy for smaller tumors and 12 Gy for larger tumors (usually $>2 \mathrm{~cm}$ in maximum extracanalicular diameter). If hearing has been lost, then $13 \mathrm{~Gy}$ is reasonable. If prior surgery has been performed and the patient has facial weakness and hearing loss, then $14 \mathrm{~Gy}$ can be considered. Using these concepts, we have reduced the incidence of delayed mild facial neuropathy to $<1 \%$.

Although there is no consensus on the dose tolerance of the cochlea, semicircular canals, or other adjacent structures, there is emerging evidence that these structures may be important to consider in dose planning (Fig. 2). These structures are best identified with long relaxation time MR sequences. Linskey et al. ${ }^{10}$ noted that the dose typically delivered to the middle ear was low, but that the inner ear and infratemporal facial nerve doses must be planned carefully. Thomas et al. ${ }^{16}$ found that the radiation dose received by the cochlea was strongly predictive of hearing deterioration after fractionated radiotherapy. Beegle et al. ${ }^{1}$ concluded that the dose was more important than conformality in the prevention of facial or trigeminal nerve deficits. Hearing outcomes were not reported. Fractionated radiotherapy, with or without stereotactic assistance (that is, SRT), has been used for cranial base tumors in an attempt to maintain cranial nerve function. The technique is not standardized, with different centers using between 3 and 30 fractions and individual doses varying between 1.8 and 7 Gy. In addition, tumor volume is crucial, and some have advocated SRT for larger-volume tumors with worse tumor control results and a higher incidence of subsequent hydrocephalus. It is clear that with SRT, target conformality and selectivity are reduced. At present, there are no long-term data to substantiate the role of SRT over SRS for appropriately selected patients.

Other cranial nerves appear to be more resistant to radiosurgical effects. When tumor margin doses of 12-14 Gy are used, deficits related to the vagal, glossopharyngeal, or hypoglossal nerves are rare. In recently evaluated series of trigeminal schwannomas and schwannomas of the oculomotor, trochlear, or abducent nerves, this dose range appears to be safe and is associated with the desired tumor response.

\section{References}

1. Beegle RD, Friedman WA, Bova FJ: Effect of treatment plan quality on outcomes after radiosurgery for vestibular schwannoma. J Neurosurg 107:913-916, 2007

2. Flickinger JC: An integrated logistic formula for prediction of complications from radiosurgery. Int J Radiat Oncol Biol Phys 17:879-885, 1989

3. Flickinger JC, Kondziolka D, Niranjan A, Maitz A, Voynov G, Lunsford LD: Acoustic neuroma radiosurgery with marginal tumor doses of 12 to 13 Gy. Int J Radiat Oncol Biol Phys 60: 225-230, 2004

4. Jackson IM, Norén G: Role of gamma knife therapy in the management of pituitary tumors. Endocrinol Metab Clin North Am 28:133-142, 1999

5. Kondziolka D, Mathieu D, Lunsford LD, Martin JJ, Madhok R, Niranjan A, et al: Radiosurgery as definitive management of meningiomas. Neurosurgery 62:53-60, 2008

6. Laws ER Jr, Vance ML: Radiosurgery for pituitary tumors and craniopharyngiomas. Neurosurg Clin N Am 10:327-336, 1999

7. Leber KA, Berglöff J, Pendl G: Dose-response tolerance of the visual pathways and cranial nerves of the cavernous sinus to stereotactic radiosurgery. J Neurosurg 88:43-50, 1998

8. Lee JY, Niranjan A, McInerney J, Kondziolka D, Flickinger JC, Lunsford LD: Stereotactic radiosurgery providing long-term tumor control of cavernous sinus meningiomas. J Neurosurg 97: 65-72, 2002

9. Lim YJ, Leem W, Park JT, Kim TS, Rhee BA, Kim GK: Cerebral infarction with ICA occlusion after Gamma Knife radiosurgery for pituitary adenoma: a case report. Stereotact Funct Neurosurg 72 (1 Suppl):132-139, 1999

10. Linskey ME, Johnstone PA, O’Leary M, Goetsch S: Radiation exposure of normal temporal bone structures during stereotacti- 
cally guided gamma knife surgery for vestibular schwannomas. J Neurosurg 98:800-806, 2003

11. Lunsford LD, Kondziolka D, Niranjan A, Flickinger JC, Maitz A: Concepts of conformality and selectivity in acoustic tumor radiosurgery. Radiosurgery 6:98-107, 2006

12. Morita A, Coffey RJ, Foote RL, Schiff D, Gorman D: Risk of injury to cranial nerves after gamma knife radiosurgery for skull base meningiomas: experience in 88 patients. J Neurosurg 90: 42-49, 1999

13. Pollock BE, Kondziolka D, Flickinger JC, Maitz A, Lunsford LD: Preservation of cranial nerve function after radiosurgery for nonacoustic schwannomas. Neurosurgery 33:597-601, 1993

14. Sheehan JM, Vance ML, Sheehan JP, Ellegala DB, Laws ER Jr: Radiosurgery for Cushing's disease after failed transsphenoidal surgery. J Neurosurg 93:738-742, 2000
15. Tishler RB, Loeffler JS, Lunsford LD, Duma C, Alexander E III, Koog, HM et al: Tolerance of cranial nerves of the cavernous sinus to radiosurgery. Int J Radiat Oncol Biol Phys 27:215-221, 1993

16. Thomas C, Di Maio S, Ma R, Vollans E, Chu C, Clark B, et al: Hearing preservation following fractionated stereotactic radiotherapy for vestibular schwannomas: prognostic implications of cochlear dose. J Neurosurg 107:917-926, 2007

Manuscript submitted March 24, 2008.

Accepted March 24, 2008.

Address correspondence to: Douglas Kondziolka, M.D., Suite B400, University of Pittsburgh Medical Center, 200 Lothrop Street, Pittsburgh, Pennsylvania 15213. kondziolkads@upmc.edu. 